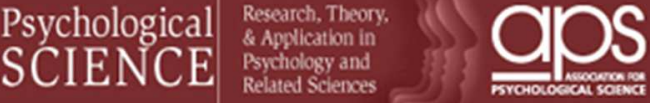

\section{Fear of holes}

\begin{tabular}{|r|l|}
\hline Journal: & Psychological Science \\
\hline Manuscript ID: & PSCI-12-1444.R2 \\
\hline Manuscript Type: & Research article \\
\hline Date Submitted by the Author: & n/a \\
\hline Complete List of Authors: & $\begin{array}{l}\text { Cole, Geoff; University of Essex, Centre for Brain Science } \\
\text { Wilkins, Arnold; University of Essex, Centre for Brain Science }\end{array}$ \\
\hline Keywords: & Threat, Fear, Evolutionary Psychology, Vision, Avoidance \\
\hline \multicolumn{2}{|l}{} \\
\hline
\end{tabular}

SCHOLARONE $^{m}$

Manuscripts 


\title{
Fear of holes
}

\author{
Geoff G Cole and Arnold J Wilkins
}

University of Essex

Colchester CO4 3SQ

Keywords: phobia, trypophobia, holes, evolution, visual stress, aposematism

\author{
Address for correspondence \\ Arnold Wilkins \\ University of Essex \\ Colchester CO43SQ
}


Abstract

Phobias are usually described as an irrational and persistent fear of certain objects or situations, and causes of such fears are difficult to identify. We describe an unusual but common phobia (trypophobia), hitherto unreported in the scientific literature, in which sufferers are averse to images of holes. We performed a spectral analysis on a variety of images that induce trypophobia and found that the stimuli had a spectral composition typically associated with uncomfortable visual images, namely high contrast energy at midrange spatial frequencies. Critically, we found that a range of potentially dangerous animals also possess this spectral characteristic. We argue that although sufferers are not conscious of the association, the phobia arises in part because the inducing stimuli share basic visual characteristics with those of dangerous organisms, characteristics that are low-level, easily computed, and therefore facilitate a rapid non-conscious response. 


\section{Introduction}

According to the Diagnostic and Statistical Manual of Mental Disorders (DSM), a phobia can be defined as a marked and persistent fear of a specific object or situation which invariably provokes anxiety. Furthermore, the individual may recognise that the fear is excessive and unreasonable. Although setting out criteria for such aversions is relatively easy, identifying the etiology of the fear is difficult. Indeed, isolating cause has been one of the main challenges of phobia research. For instance, there have been accounts of phobia based on evolutionary principles (Marks \& Nesse, 1994), classical conditioning (e.g., Merckelbach \& Muris, 1997) and the role of thoughts and beliefs about objects and situations (e.g., Hertel \& Brozovich, 2010).

Such theories of phobia acquisition can have difficulty explaining many phobias. A case in point, and central to this article, is trypophobia; the 'fear of holes'. Sufferers report aversion to visual stimuli comprising particular configurations of holes. The stimuli are usually clusters of holes of any variety that are almost always innocuous and seemingly pose no threat. Although no peer-reviewed papers currently exist concerning the phenomenon, the internet presence is greater than that of several more widely recognised phobias, and there are a number of internet-based support groups, including a Facebook site, where people provide testimonials. It is clear from these descriptions that for many people trypophobia affects their everyday lives and can be quite debilitating. For instance, one sufferer reports: "(I) can't really face small, irregularly or asymmetrically placed holes, they make me like, throw up in my mouth, cry a little bit, and shake all over, deeply." The image most often reported as inducing the phobia is the seed head of the lotus flower (see Figure 1). Other examples include soap bubbles and the holes in aerated chocolate. To obtain an initial estimate of how common trypophobia is, the authors asked 91 male and 195 female adults whose age ranged 
from approximately 18 to 55 , to view an image of the lotus seed head. The participants indicated whether the image was 'uncomfortable or even repulsive to look at'. Ten males $(11 \%)$ and 36 females (18\%) reported aversion.

\section{INSERT FIGURE 1 ABOUT HERE}

Importantly, sufferers report that it is the visual percept that is particularly aversive. This can be contrasted with, for instance, an aversion to cats in which a person with ailurophobia will be uncomfortable in the presence of a cat even if it is not visible. Furthermore, trypophobia is seemingly increased if the holes occur on human skin. It is only in this respect that the phobia involves any reference to the semantics of the image. The visual nature of trypophobia provides a clue as to its cause. Aversion and discomfort when viewing certain geometric patterns has been known for many years (Wilkins et al., 1984). Motivated by sporadic media reports over the past four decades of public artworks inducing migraine, Fernandez and Wilkins (2009) examined the spectral characteristics of images that induce aversion. Any visual image can be analysed with respect to its fundamental visual properties. For instance, chromatic and brightness (luminance) contrasts can be computed at any point in a scene. A major property of the visual world is luminance contrast, which can be derived at various spatial scales. An image can be constructed from Fourier components consisting of luminance varying sinusoidally at different spatial frequencies, phases, contrasts and orientations. One of the fundamental properties of a visual scene is the relationship between luminance contrast and spatial frequency. In scenes from nature the spatial frequency and contrast of the components are related such that contrast increases as spatial frequency decreases. When log contrast energy is plotted against log spatial frequency a straight line with a slope close to -1 is typically found (Field \& Brady, 1997). The image is then scale 
invariant: the complexity of the scene is independent of spatial scale. In other words, the natural visual world has a characteristic visual property revealed with a spectral analysis. However, this particular property is not found in images that are uncomfortable to look at. Fernandez and Wilkins asked participants to rate discomfort from a wide variety of images including paintings, photographs and meaningless images created from random noise. Images rated as being particularly uncomfortable possessed Fourier spectra with an excess of contrast energy at mid-range spatial frequencies relative to that expected elsewhere in the spectrum. Thus, uncomfortable images do not possess the characteristic visual property in which contrast amplitude decreases linearly with increasing spatial frequency; rather, they tend to have relatively large contrast at mid-range spatial frequencies. The discomfort is dependent on amplitude rather than phase. These findings have since been confirmed by O'Hare and Hibbard (2011).

Given the knowledge that images associated with aversion have a characteristic spectral composition, the current work examines whether trypophobia may arise partly because the inducing images possess this unusual feature, that is, relative excess of contrast energy at mid-range spatial frequencies. We performed a spectral analysis on a range of trypohobiainducing images and compared them with control images of holes that do not induce trypophobia. To pre-empt part of our results, we find that images inducing trypophobia do not possess the characteristic property of natural images; they have excess contrast energy at mid-range spatial frequencies. We further show that images of potentially dangerous animals also possess this unusual feature. We will therefore argue that trypophobia arises because the inducing images and objects share a simple visual property with objects that are potentially dangerous. 


\section{Experiment 1: Analysis of trypophobic images}

Image acquisition and spectral analysis.

Images were obtained from the typophobia website, www.trypophobia.com. They included images of a lotus seed pod and a wide range of other images of clusters of holes, 76 images in total. We took the first 76 images presented without prejudice and none were of the skin lesion type. A Google search for "images of holes" provided a set of 76 control images of holes that were not exhibited on www.trypophobia.com as associated with trypophobia. Using Matlab, the images were cropped to give the largest central square image, resized to 512 x 512 pixels (using nearest neighbour algorithm), rendered in grey level with the rgb2gray function (0-255) and normalised so that the mean grey level was 125 and the standard deviation 25. A Hanning window was applied, which reduced the contrast to zero at the periphery so as to remove edge effects. The FFT provided an amplitude spectrum in two dimensions and this matrix was sampled using a set of annular masks that summed the energy over all orientations. The internal and external dimensions of the annuli were such as to sum the energy in bins of equal size on a logarithmic scale of spatial frequency, each bin differing from its neighbour by a factor of square root of 2 . The four lowest spatial frequency bins were removed from analysis owing to the effects of the Hanning window.

\section{INSERT FIGURE 2 ABOUT HERE}

Figure 2 shows the power spectrum of the control images and those obtained from the trypophobia site. Overall a power function (linear on log-log axes) accounted for more than $97 \%$ of the variance, a good fit to the prediction for natural images. The percentage variance 
explained by the linear fit to the average spectra for the trypophobic images of holes $(95.7 \%)$ was significantly less than for the non-trypophobic images of holes $(97.9 \%), t(121)=3.31$, $\mathrm{p}<0.001$. These findings are consistent with a greater energy at mid and high spatial frequencies in the trypophobic images. In Figure 2 the spatial frequency has been expressed in cycles per image (cpi). Using a Bonferroni correction for 12 paired comparisons of $p=0.0043$, the difference in power between the two functions is significant for spatial frequencies in the range 45-181cpi. Most photographic images subtend 10-30 degrees arc, so from the viewpoint of the camera, the excess in contrast energy ranged from a minimum of 45 cycles per image divided by 30 degrees per image (i.e., 1.5 cycles per degree) to a maximum of $181 / 10$ (i.e., 18) cpd. Objects are usually photographed so that they fit most of the frame, and in consequence, small objects may be photographed from distances less than those from which they are typically viewed, and the reverse holds for large objects. Fernandez and Wilkins (2009) showed that the range of spatial frequencies for which an excess energy can be expected in uncomfortable images is $1-8 \mathrm{cpd}$; a range of a factor of 8 . Given this large range it seems likely that, even allowing for the typical viewing distance of small and large objects, this critical spatial frequency range expressed in cycles per degree is within the range for which the two curves are maximally and significantly divergent. In sum, Experiment 1 has shown that trypophobic images have a visual property not usually possessed by natural images; they have relatively high contrast at mid-range spatial frequencies.

\section{Experiment 2: Generality of the aversion}

Our second study examined whether aversion to trypophobic images extends across the general population. Fifty images from the trypophobia website and 50 images of holes obtained from a Google search were presented in random order as a PowerPoint presentation to 20 undergraduate students at the University of Essex, none of whom reported being 
trypophobic. The students were asked to rate any discomfort from the images on a scale from -5 representing maximum discomfort to +5 representing maximum comfort. The mean rating for the trypophobic images was -0.42 and for the control images $+0.53, \mathrm{t}(49)=4.67, \mathrm{p}<0.0001$. Evidently trypophobic images are uncomfortable not simply for a minority of individuals who profess to a phobia, but also more generally.

\section{Experiment 3: Analysis of images of poisonous animals}

In our third study we attempted to identify the cause of trypophobia by assessing the spectral composition of poisonous animals. This was motivated by an individual who reported a fear of holes and told the authors that certain animals also induce aversion (e.g., Blue Ringed Octopus). The common aspect of the animals seemed to be that they are highly poisonous. We obtained images of animals that in a large number of sources on the web have been listed as "the 10 most poisonous animals". They are extremely poisonous to humans and consequently are commonly considered dangerous. The 10 are: Blue Ringed Octopus, Box Jellyfish, Brazillian Wandering Spider, Death Stalker Scorpion, Inland Taipan snake, King Cobra snake, Marbled Cone Snail, Poison Dart Frog, Puffer Fish, and the Stone fish. Ten different images of each species were obtained from the web and are available as supplementary material. As with the typophobic images we analysed the first 10 images that a Google image search generated without prejudice, the only constraint as to selection being that the size of the image exceeded 300 pixels on its smaller dimension. The images were photographs of the individual animals, on a variety of backgrounds, mainly natural. In most photographs the animal was close to the centre of the image and in longer dimension occupied more than 50\%. The images had JPEG format, which involves lossy compression. To control for any artifacts such compression might introduce, the images were compared with images of otherwise similar but non-poisonous species, sourced and prepared identically. These were octopus of various non-poisonous species, non-poisonous jellyfish, 
spiders, crabs, non-venomous snakes, non-toxic frogs, edible snails, edible fish.

\section{INSERT FIGURE 3 ABOUT HERE}

Figure 3 shows the log contrast energy as a function of log spatial frequency for the images of the poisonous and non-poisonous animals. Overall a power function (linear on log-log axes) accounted for more than $99 \%$ of the variance, again a good fit to the prediction for natural images. The percentage variance explained by the linear fit to the average log spectra for the 10 highly poisonous animals we selected (99.1\%) was less than for the 10 control animals $(99.6 \%), \mathrm{t}(9)=2.58, \mathrm{p}=0.03$. Notwithstanding the normalisation of all images in terms of pixel mean and variance, there was $15 \%$ more contrast energy at mid-range spatial frequencies in the images of the poisonous animals ( $<<0.05$, between 16 and $32 \mathrm{cpi}$ ); the size of this difference is masked by the logarithmic scale in Figure 3. The excess was obtained not only in the grey level images but also in the images formed from the $\mathrm{R} G$ and $\mathrm{B}$ pixels taken separately, suggesting that the excess was not dependent on a particular coloration, or indeed a particular spectral sensitivity. In sum, these results show that the highly poisonous animals possess a spectral feature similar to that of the trypophobic images.

\section{Experiment 4: Snakes and spiders}

Some of the more common phobias are those of snakes and spiders and many individuals are unable to look at images of these animals without aversion (e.g. Ohman, Flykt \& Esteves, 2001). This is the case even in countries where spiders are not venomous and present no threat. Furthermore, a number of standard behavioural measures have been used to assess the processing priority given to such objects. For instance, in a typical attention task, observers are required to detect the presence of a target item as quickly as possible. Relatively short response times are usually taken as a marker of cognitive biases towards particular stimuli 
(e.g., Crundall, Cole, \& Galpin, 2007). Both adults and young children have repeatedly been found to detect snakes more rapidly than other kinds of stimuli. Lobue and Deloache (2011) measured the time taken to detect images of snakes and frogs by young children. They found that the snakes were more rapidly detected and that it was the coiled body shape rather than their sometimes colourful markings that was largely responsible for the conspicuity. Such a spectral power distribution is likely to be conspicuous because it differs from the spectral energy most pervasive in nature.

Motivated by this prior work we analysed images of snakes and spiders. Twenty images of a snake and 20 of a spider having a smaller dimension of at least 300 pixels were sourced from Google, again in order of acquisition and without prejudice. They were processed as in Experiment 1. Both spectra were curved downward as in Figure 3. A power function accounted for an average of $98.5 \%(\mathrm{SD}=1.1 \%)$ of the variance of the spectra of the snake images and $98.6 \%(\mathrm{SD}=1.0 \%)$ of the spider images. These figures are substantially lower than for the images of the control animals in Experiment 3. Thus, as with the poisonous animals analysed in Experiment 3, images of snakes and spiders do not show the usual linear relationship of log contrast energy to log spatial frequency.

\section{General Discussion}

We have found that images responsible for a previously undescribed but relatively common form of visual phobia possess a property characteristic of images that are generally uncomfortable to view. They show comparatively high contrast energy at mid-range spatial frequencies. This confirms the results of Fernandez and Wilkins (2009) who found a similar property in a variety of images. Importantly, we have also found that images of animals well known to be dangerous also possess this visual property. We therefore suggest that trypophobia arises because the inducing stimuli share a core spectral feature with such 
organisms: a feature that does not reach conscious awareness. In other words, if any stimulus, such as a configuration of holes, coincidently possesses this spectral feature the stimulus may induce some form of aversion because of the survival value of such aversion.

This survival account is based on the notion that humans have been selected, via Darwinian principles, for their ability to notice poisonous organisms. The notion that phobias can be explained by an innate predisposition to fear potentially dangerous stimuli (e.g., Marks \& Nesse, 1994) is often contrasted with the view that the etiology of phobia is due to a learning process. Aversion to dangerous objects is said to have resulted in modern humans possessing an innate predisposition to develop fears of certain objects such as snakes, spiders, heights, etc. An alternative Darwinian explanation is that the ability to effectively process dangerous stimuli evolved before humans originated. Possessing patterns as a warning of unpalatability (i.e., aposmatism) is a well established method of defence (e.g., Santos, Coloma, \& Cannatella, 2003). Such patterns tend to be characterised by their high contrasting colours at mid-range spatial frequencies. Furthermore it is widely accepted that the visual system has been selected for its ability to orient attention to the location of a new object in the visual field (e.g., Abrams \& Christ, 2003; Cole \& Kuhn, 2009; 2010). However, conscious recognition of an object is a slow process taking up to 350 milliseconds (Johnson \& Olshausen, 2005). Responding to a potential threat such as a snake on the basis of relatively slow conscious perception could be costly to an organism. An alternative more effective detection/avoidance strategy might be to respond to the presence of an object via an 'early' fast-acting visual mechanism based on a simple feature that is common to most dangerous animals. In addition to motion (e.g., Cole, Heywood, Kentridge, Fairholm, \& Cowey, 2003; Skarratt, Cole, \& Gellatly, 2009), the computation of contrasts at various spatial scales provides just such a low-level mechanism. Support for this idea comes from other work that 
has examined threat related stimuli with respect to low-level features. For instance, Bannerman, Hibbard, Chalmers, and Sahraie (2012) required observers to make a saccade to happy, fearful or neutral faces that had been filtered to comprise predominantly low, high, or broad spatial frequencies. Amongst a number of effects, the authors reported that at low spatial frequencies fearful faces showed the fastest saccadic responses. In contrast, there was no difference in mean latency between any emotions for higher spatial frequencies. Similarly, Vuilleumier, Armony, Driver, and Dolan (2003) showed that amygdala activity was greater for the processing of fearful expressions for faces containing low spatial as opposed to high spatial frequencies.

Given the large number of images that possess an excess of energy at mid-range spatial frequencies (Fernandez \& Wilkins, 2008), it is most unlikely that this spectral feature is a sufficient condition for phobia, even though it is associated with aversion. Nevertheless, it may prove possible to offer treatment by progressive spatial filtering of the offensive images. It is, of course, still unknown why some people develop an aversion to holes whilst others do not. This issue is common to all explanations of phobia; some people who have not suffered an animal bite become phobic to dogs whilst others who have suffered such a bite do not become phobic. However our results from Experiment 2 do suggest that non-trypophobic individuals are sensitive to the inducing stimuli in that they perceive trypophobic images of holes to be more aversive to look at than non-trypophobic images of holes. Perhaps the condition is a matter of degree, an exaggeration of a normal tendency. Finally, although the aversion has become known as the 'fear of holes' our data reveal that one essential characteristic that induces the aversion is a particular spectral property, a property often associated with relatively high contrast material at mid-range spatial frequencies and not necessarily involving holes. 
References

Abrams, R. A., \& Christ, S. E. (2003). Motion onset captures attention. Psychological Science, 14, 427-432.

Bannerman, R.L., Hibbard, P.B., Chalmers, K. \& Sahraie, A. (2012). Saccadic latency is modulated by emotional content of spatially filtered face stimuli. Emotion (in press)

Bannerman, R. L., Milders, M., de Gelder, B., \& Sahraie, A. (2009). Orienting to threat: Faster localization of fearful facial expressions and body postures revealed by saccadic eye movements. Proceedings of the Royal Society of London B, 276; 1635-1641.

Bolton, D., Eley, T., O’Connor, T., Perrin, S., Rabe-Hesketh, S., Rijsdijk, F., \& Smith, P. (2006). Prevalence and genetic and environmental influences on anxiety disorders in 6-yearold twins. Psychological Medicine, 36; 335-344.

Cole, G. G., \& Kuhn, G. (2009). Appearance matters: Attentional orienting by new objects in the precuing paradigm. Visual Cognition, 17, 755-776.

Cole, G. G., \& Kuhn, G. (2010). Attentional capture by object appearance and disappearance. Quarterly Journal of Experimental Psychology, 63, 147-159.

Cole, G. G., Heywood, C. A., Kentridge, R. W., Fairholm, I. \& Cowey, A. (2003). Attentional capture by colour and motion in cerebral achromatopsia. Neuropsychologia, 41, 1837-1846.

Crundall, D., Cole, G. G., \& Galpin, A. (2007). Object-based attention is mediated by collinearity of targets. Quarterly Journal of Experimental Psychology, 60, 137-153.

Fernandez, D. \& Wilkins A.J. (2008). Uncomfortable images in art and nature. Perception, 37(7), 10981113.

Field, D.J. \& Brady, N. (1997). Visual sensitivity, blur and the sources of variability in the amplitude spectra of natural scenes. Vision Research, 37(23), 3367-3383.

Hertel, P. T., \& Brozovich, F. (2010). Cognitive habits and memory distortions in anxiety and depression. Current Directions in Psychological Science, 19, 155-160. 
Johnson, J. S., \& Olshausen, B. A. (2003). Timecourse of neural signatures of object recognition. Journal of Vision, 3, 499-512.

Marks, I. M., \& Nesse, R. M. (1994). Fear and fitness: An evolutionary analysis of anxiety disorders. Ethology and Sociobiology, 15, 247-261.

Merckelbach H., \& Muris, P. (1997). The etiology of childhood spider phobia. Behaviour Research and Therapy, 35, 1031-1034.

O’Hare L \& Hibbard P.B. (2011). Spatial frequency and visual discomfort. Vision Research. 51(15), 1767-1777.

Ohman, A, Flykt, A \& Esteves, F. (2001). Emotion drives attention: detecting the snake in the grass. Journal of Experimental Psychology: General 130(3), 466-478.

Skarratt, P., \& Cole, G. G., \& Gellatly, A.R.H. (2009). Prioritisation of looming and receding objects: Equal slopes, different intercepts. Attention, Perception \& Psychophysics. 71, 964970.

Santos, J.C., Coloma L.A., \& Cannatella D.C., 2003. Multiple, recurring origins of aposematism and diet specialization in poison frogs. Proceedings of the National Academy of Sciences. 100, 12792-12797.

Vuilleumier, P., Armony, J.L., Driver, J., Dolan, R.J. (2003). Distinct spatial frequency sensitivities for processing faces and emotional expressions. Nature Neuroscience, 6(6), 62431.

Wilkins, A.J., Nimmo-Smith, M.I., Tait, A., McManus, C., Della Sala, S., Tilley, A., Arnold, K., Barrie, M., \& Scott, S. (1984) A neurological basis for visual discomfort. Brain, 107, 989-1017. 


\section{Figure Legends}

Figure 1. Lotus seed head.

Figure 2. Power spectra of typophobic (broken line) and control (solid line) images of holes.

Figure 3. Power spectra of images of poisonous (broken line) and non-poisonous (solid line) animals. Note that the difference in power at mid-range spatial frequencies is more than $15 \%$. 


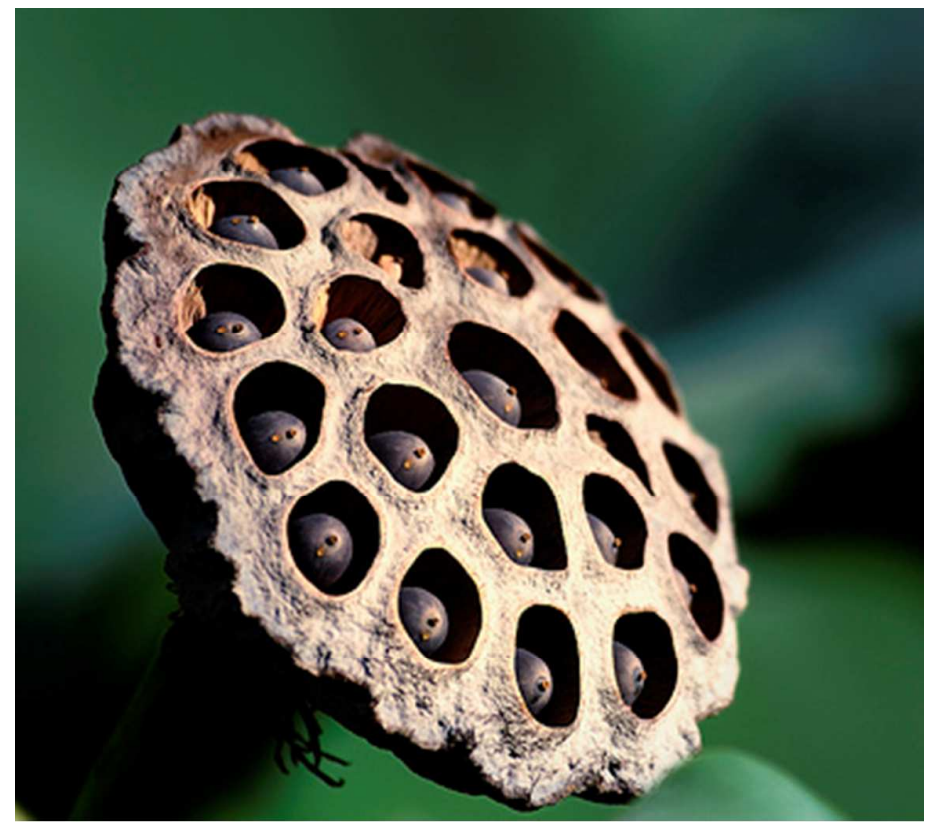

Figure 1 


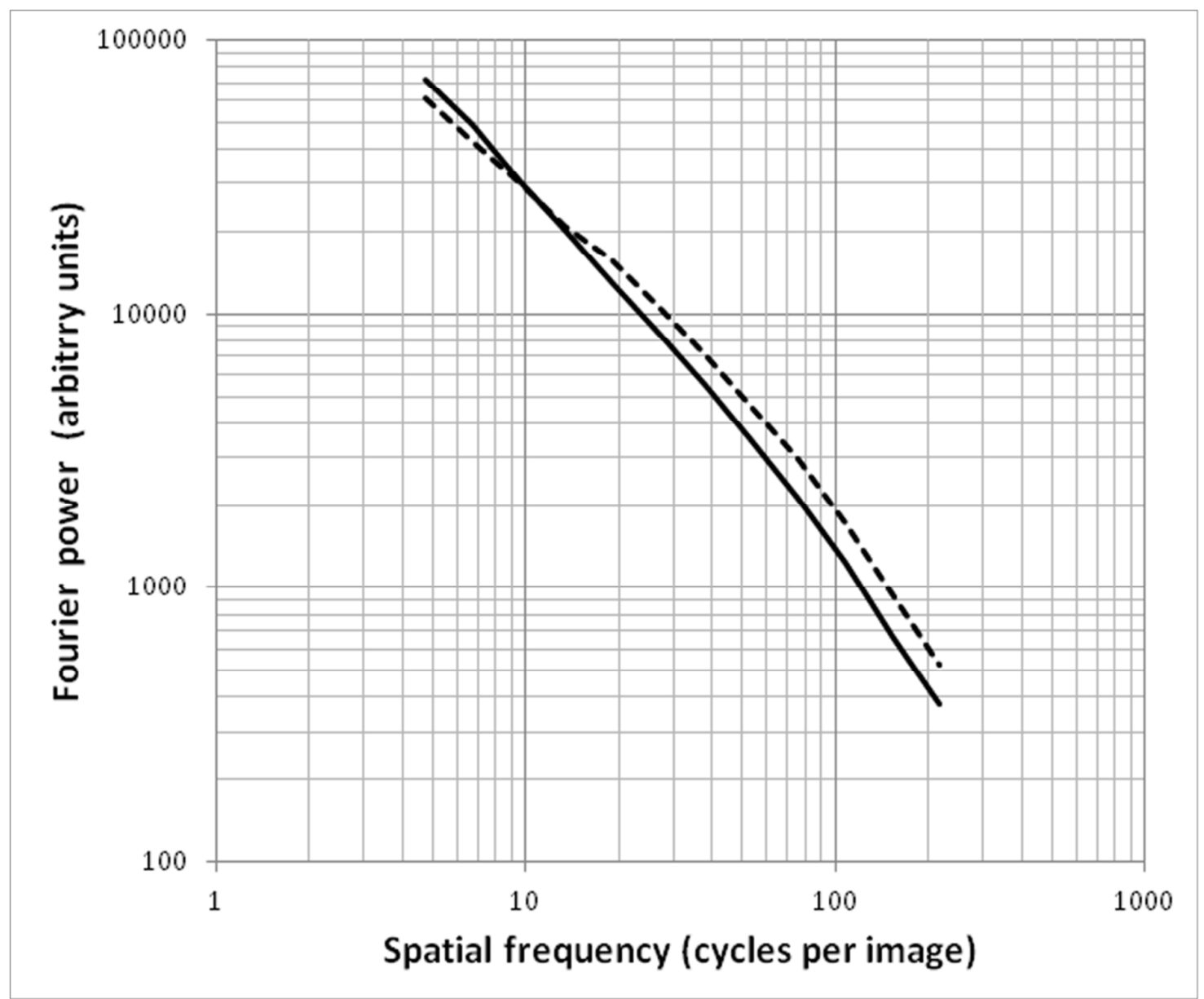

Figure 2 


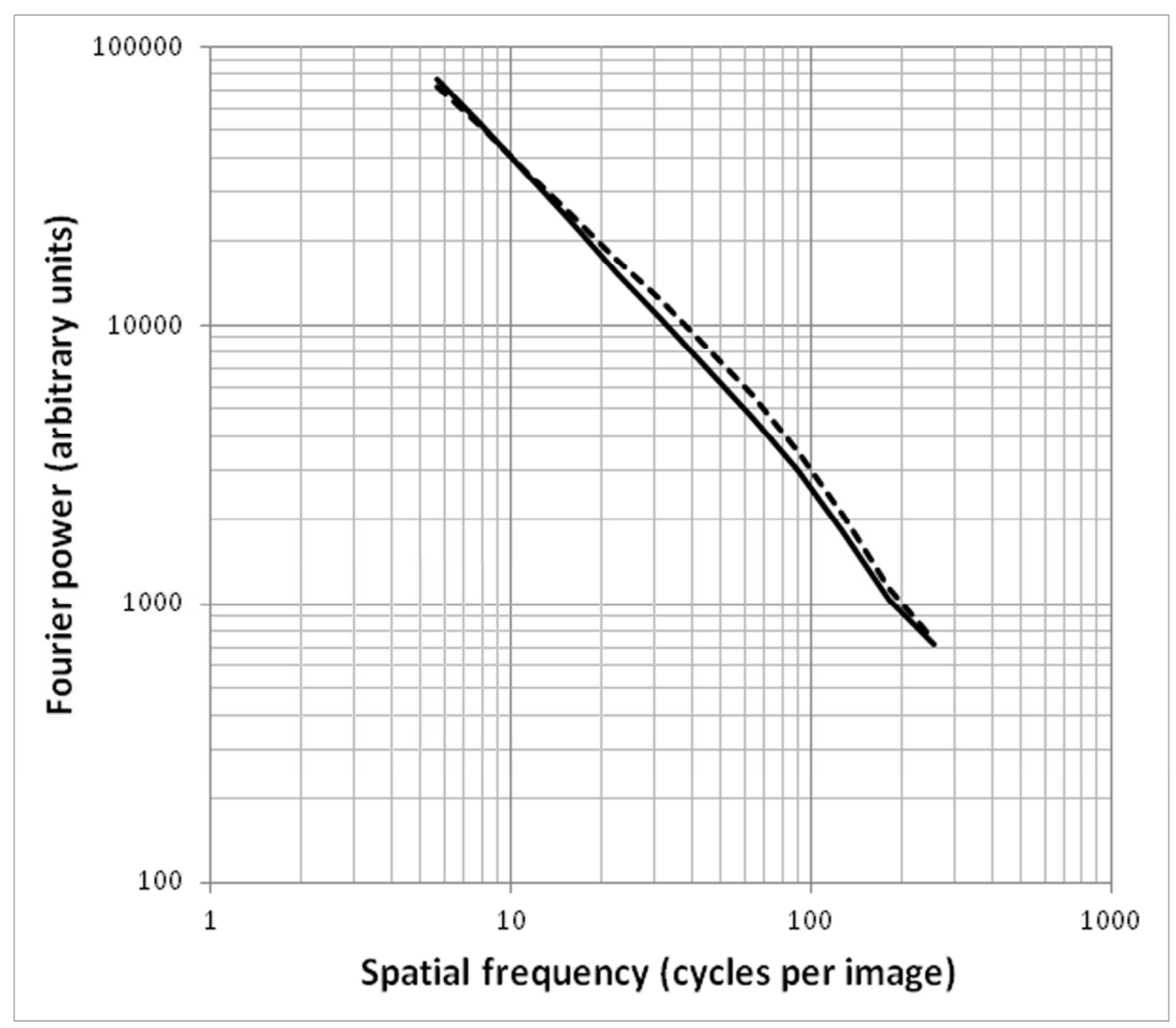

Figure 3 